Gynaecologia 1955;139:236

\title{
Problèmes de stérilité vus par le gynécologue-praticien
}

D.

\author{
Stucki
}

\section{Porrentruy}

(Paraîtra prochainernent in extenso dans « Praxis »)

Presentation de 120 ca.s de stérilité primaire étudiés durant les années 1950-1953 quant à leur étiologie, les méthodes d'examen les plus employees et les plus nécessaires, les résultats des traite-rnents divers, tant medical que chirurgical. Comparaisons statis-tiques à $\Gamma$ aide de tabelles entre le traitement de la stérilité masculine et feminine.

Etterich, M. und M. Mall: Gynaecologia 13V, 236, 1955

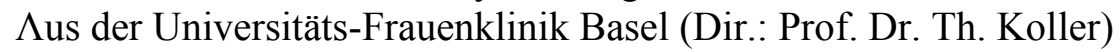

Sklerodermie und Schwangerschaft

Von M. ETTERICH und M. MALL

Wenn wir über einen Eall von Sklerodermie und Schwangerschaft berichten so gesehieht dies nicht

um einen genauen intern-medizi-nischen oder pathologisch-anatomischen Querschnitt dieses seltenen Krankheitsbildes zu übermitteln. Die Zeit dazu ware viel zu kurz und eine Versammlung von Gynäkologen wahrscheinlich auch nicht gerade das interessierteste Gremium für ein so ausgesprochen intern- 